\title{
A sensitive magnetic field sensor using BPSCCO thick film
}

\author{
S VIJAY SRINIVAS, ABHIJIT RAY and T K DEY* \\ Cryogenic Engineering Centre, Indian Institute of Technology, Kharagpur 721 302, India
}

MS received 15 December 2000; revised 29 May 2001

\begin{abstract}
A highly sensitive magnetic sensor operating at liquid nitrogen temperature and based on BPSCCO screen-printed thick film, is reported. The sensor resistance for an applied magnetic field of $100 \times 10^{-4} \mathrm{~T}$ (100 gauss) exhibits an increase by $360 \%$ of its value in zero field at $77.4 \mathrm{~K}$. The performance of the sensor in presence of magnetic field, the hysteretic features and the effect of thermal cycling, has been discussed.
\end{abstract}

Keywords. High temperature superconductors; thick film; magnetic sensor.

\section{Introduction}

Magnetic sensors are important for their diverse potential applications, viz. from simple compass based navigation systems to ultra sophisticated SQUID devices that probe the invisible human biological activities. Several magnetic sensors based on widely different principle of operation have been developed (Dibbern and Petersen 1983; Lenz 1990; Ripka 1992; Heremans 1993). Each of them has its own advantages and disadvantages. Discovery of high temperature superconductors have opened up newer possibilities for their applications in the fabrication of magnetic shields (Itoh et al 1997), magnetic bearings (Itoh et al 1989) as well as various types of detectors (Zheng et al 1990; Wise et al 1993) and sensors (Villegier et al 1991). Apart from the most sensitive of all existing magnetic sensors (the SQUIDs), the magnetic sensors based on the second harmonic response of magnetic susceptibility (Gallop et al 1989; Gielisse et al 1991; Khare et al 1997; Dey et al 1999), those based on the sharp jump of trapped magnetic flux (Rostami et al 1998) as well as, on the microwave properties (Kirschner et al 1994) of bulk and thin films of ceramic superconductors have been proposed. Of these the magnetic field sensors based on the $2 \mathrm{f}$ response of weak links present in high temperature superconductors are capable of detecting of field in the range from $10^{-4} \mathrm{~T}$ to $10^{-8} \mathrm{~T}$ and they are expected to find applications for non destructive evaluation of cracks in ferromagnetic plates etc (Buckley et al 1991).

In the present communication, we report the performance of a simple inexpensive yet sensitive magnetic field sensor fabricated using BPSCCO screen printed thick film. In this application, BPSCCO thick film sensors are prepared in such a way so as to retain a small resis-

\footnotetext{
*Author for correspondence
}

tance $\left(R_{77}\right)$ at the boiling point of liquid nitrogen $(77.4 \mathrm{~K})$. This residual resistance of the sensor at $77.4 \mathrm{~K}$ has been found to be highly sensitive to the magnitude of the external d.c. magnetic field. Influence of the preparation condition on the temperature dependence of the electrical resistivity of the sensors, the effect of the external d.c. magnetic field $\left(H_{\text {d.c. }}\right)$ on the sensor response, their hysteresis characteristics and effect of thermal cycling has been discussed.

\section{Experimental}

Thick films of $(\mathrm{Bi}, \mathrm{Pb}) 2223$ superconductor were prepared from fine grained $(5 \mu)$ precursor powder of $\mathrm{Bi}_{1 \cdot 6} \mathrm{~Pb}_{0.4} \mathrm{Sr}_{2} \mathrm{Ca}_{2} \mathrm{Cu}_{3} \mathrm{O}_{x}$ procured from M/s Can Superconductors, Czech Republic. The superconducting ( $\mathrm{Bi}, \mathrm{Pb}$ ) 2223 powder was thoroughly mixed with an organic binder (cyclohexane) to form a paste of appropriate viscosity. The prepared paste was then screen printed on a polished $\mathrm{MgO}$ single crystal (100) substrate using fine nylon screen. The screen-printed film was first dried at $200 \mathrm{C}$ for $2 \mathrm{~h}$ to remove the organic binder. The dried film was then heat treated in air at $880 \mathrm{C}$ for different lengths of time (table 1) and was subsequently furnace cooled. The prepared films $(10 \times 5 \mathrm{~mm})$ were fairly uniform and typical film thickness varied between 200 and $250 \mu \mathrm{m}$. Figure 1 shows the XRD of $\mathrm{Bi}_{1.6} \mathrm{~Pb}_{0.4} \mathrm{Sr}_{2} \mathrm{Ca}_{2} \mathrm{Cu}_{3} \mathrm{O}_{x}$ thick film (sensor \#1), as well as, that for the precursor powder. It is apparent from figure 1 that 2223 phase constitutes the major phase in both the precursor powder and in the screen-printed films. Further, the phase composition remains unaltered after screen-printing.

Measurements of the temperature dependence of electrical resistance of the prepared thick films were performed in a helium exchange gas cryostat, positioned at the centre of a solenoid. The direction of magnetic field was perpendicular to the surface of the thick film. The 
magnetic field inside the solenoid was calibrated by a Hall sensor (Lake Shore LHGA 321). Temperature of the thick films was monitored by a calibrated Si-diode thermometer (DT 470) in combination with a digital temperature indicator (Lake Shore model 550) to a resolution of $\pm 0.01 \mathrm{~K}$. Four-probe method was adopted to measure the resistance of the thick film sensor. Current and voltage leads were attached to the sensor by a conductive silver paste. A programmable d.c. current source (Advantest model TR6142) was used to provide current through the sensor and the resistive drop across the sensor was measured using a digital nano voltmeter (Keithley Model 181) to a resolution of $\pm 0.01 \mu \mathrm{V}$.

\section{Results and discussion}

Figure $2 \mathrm{a}$ shows the temperature variation of the normalized resistance $\left(R_{\mathrm{T}} / R_{300}\right)$ for the BPSCCO thick film sensors and for a pellet prepared using the precursor powder between 300 and $77.4 \mathrm{~K}$ in the absence of external magnetic field. Significant influence of the duration of the heat treatment time on the quality of thick film is clearly evident. As is expected, the resistance of the films systematically decreases with the increase in heat treatment time. All the screen-printed films show superconducting onset close to $115 \mathrm{~K}$. However, the samples $\# 1$, \#2 and \#3 retain a small residual resistance $\left(R_{77}\right)$ of $1.46 \Omega, 3.51 \Omega$ and $4.53 \Omega$ respectively at liquid nitrogen temperature (table 1). Typical dependence of the sensor resistance at $77.4 \mathrm{~K}$ on the sensor current $\left(I_{\mathrm{s}}\right)$ for various values of external magnetic fields is plotted in figure $2 \mathrm{~b}$. Sensor resistance $\left(R_{77}\right)$ at $77.4 \mathrm{~K}$ is found to be essentially independent of $I_{\mathrm{s}}$ between $0.01 \mathrm{~mA}$ and $0.2 \mathrm{~mA}$ and it shows an increase for $I_{\mathrm{s}}$ exceeding $0.2 \mathrm{~mA}$. All the thick film magnetic sensors reported here have been characterized using a driving current of $0 \cdot 1 \mathrm{~mA}$.

The response of the sensors due to the external d.c. magnetic field $\left(H_{\text {d.c. }}\right)$ at liquid nitrogen temperature is plotted in figure 3 , where, the resistance in presence of
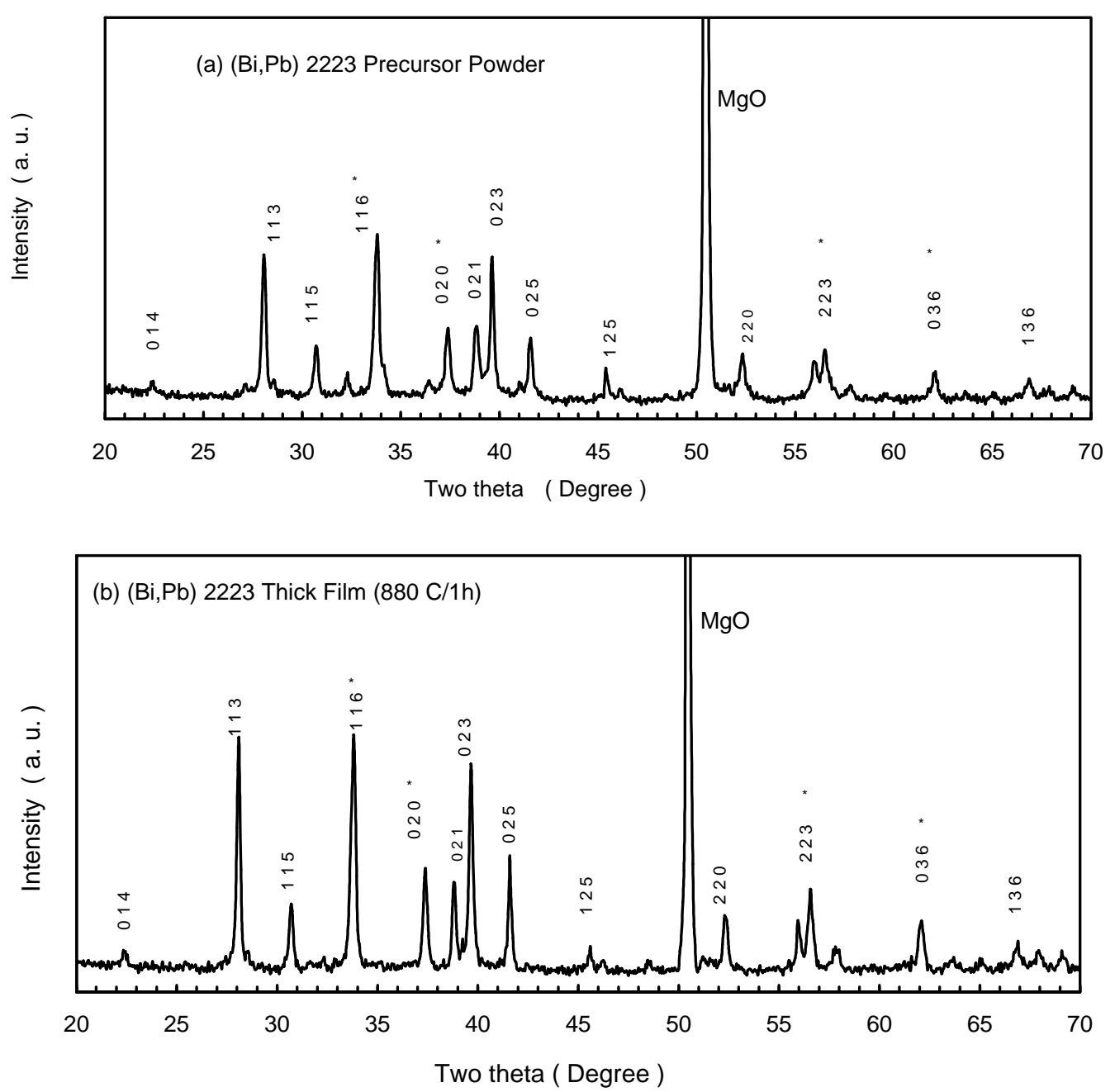

Figure 1. $\mathrm{XRD}$ for (a) $\mathrm{Bi}_{1 \cdot 6} \mathrm{~Pb}_{0.4} \mathrm{Sr}_{2} \mathrm{Ca}_{2} \mathrm{Cu}_{3} \mathrm{O}_{x}$ precursor powder and (b) for screen printed thick film (sensor \# 1). 2212 phase $(*)$. 
Table 1. Characteristics of BPSCCO thick film magnetic sensors.

\begin{tabular}{lcccc}
\hline Sensor & $\begin{array}{c}\text { Heat treatment } \\
\text { schedule }\end{array}$ & $T_{\mathrm{c}}^{\text {onset }}(\mathrm{K})$ & $R_{77}(\Omega)$ & $\begin{array}{c}R(10 \mathrm{mT}) / R_{77}(0) \\
\text { at 77 K }(\%)\end{array}$ \\
\hline$\# 1$ & $880 \mathrm{C}(1 \mathrm{~h})$ & 115 & 1.46 & 360 \\
$\# 2$ & $880 \mathrm{C}(2 \mathrm{~h})$ & 115 & 3.51 & 155 \\
$\# 3$ & $880 \mathrm{C}(4 \mathrm{~h})$ & 115 & 4.53 & 110 \\
\hline
\end{tabular}
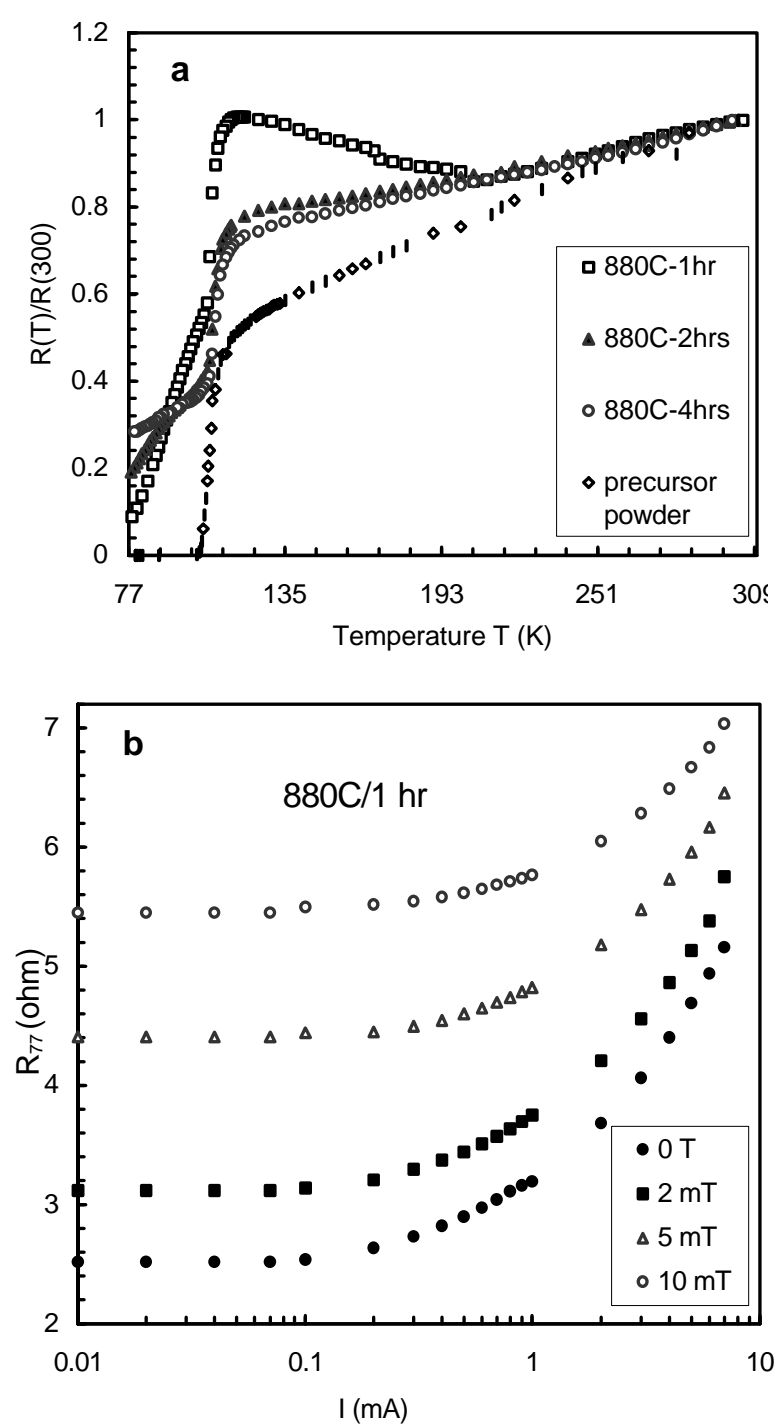

Figure 2. a. Temperature dependence of the resistance of the BPSCCO screen printed thick films and that for a pellet prepared from the precursor powder measured between $300 \mathrm{~K}$ and $77.4 \mathrm{~K}$ and $\mathbf{b}$. dependence of the sensor resistance $\left(R_{77}\right)$ at liquid nitrogen temperature as a function of the driving current $\left(I_{\mathrm{s}}\right)$ for different values of applied d.c. magnetic fields $\left(H_{\text {d.c. }}\right)$.

the magnetic field, $R\left(H_{\text {d.c. }}\right)$ has been normalized by its resistance in zero field, $R_{77}\left(H_{\text {d.c. }}=0\right)$ at $77.4 \mathrm{~K}$. A very good symmetrical response with respect to the direction of the magnetic field in $\pm 400 \times 10^{-4} \mathrm{~T}$ is clearly evident for all the sensors. It is important to note that for the

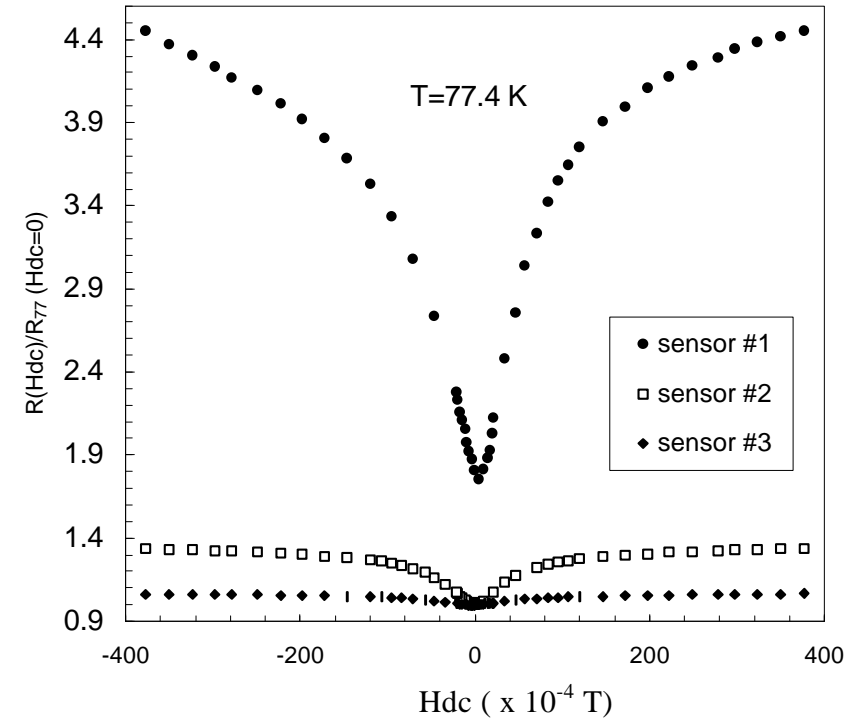

Figure 3. Sensor resistance as a function of the applied d.c. magnetic field $\left(H_{\text {d.c. }}\right)$ in the range from -40 to $+40 \mathrm{mT}$.

sensor $\# 1$ heat treated at $880 \mathrm{C}$ for $1 \mathrm{~h}, R_{77}$ at $10 \mathrm{mT}$ exhibits a very large increase of $360 \%$ over its resistance in zero magnetic field. This kind of sensitivity is much higher than the sensitivity of many semiconducting magnetoresistive sensors (Dibbern and Petersen 1983; Lenz 1990). However, the sensor response $R\left(H_{\text {d.c. }}\right) / R_{77}(0)$ is found to be substantially lower for the samples prepared with longer heat treatment time (table 1). In the low field region (viz. between 0 and $3 \mathrm{mT}$ ), the sensor response follows a relation of the type:

$$
R\left(H_{\text {d.c. }}\right)=R_{77}\left(1+\alpha H_{\text {d.c. }}^{2}\right) \text {. }
$$

The constant $\alpha$ takes the values of $0.44 \times 10^{-3}, 0.15$ $\times 10^{-3}$ and $0.02 \times 10^{-3}$ respectively for the sensors $\# 1$, \# 2, and \# 3. As shown in figure 3 , in the field range between 2 and $9 \mathrm{mT}$, the sensor's response is fairly linear. Typical hysteresis characteristics as the one shown in figure $4 \mathrm{a}$ were observed for all our BPSCCO thick film sensors for $H_{\text {d.c. }}>12 \mathrm{mT}$. However, no evidence of hysteresis was displayed for fields below $12 \mathrm{mT}$ (figure 4b). It is important to mention that no worthwhile degradation in sensor's response, or, deterioration of the film was observed after five thermal cyclings between room and liquid nitrogen temperature. However, considering the 

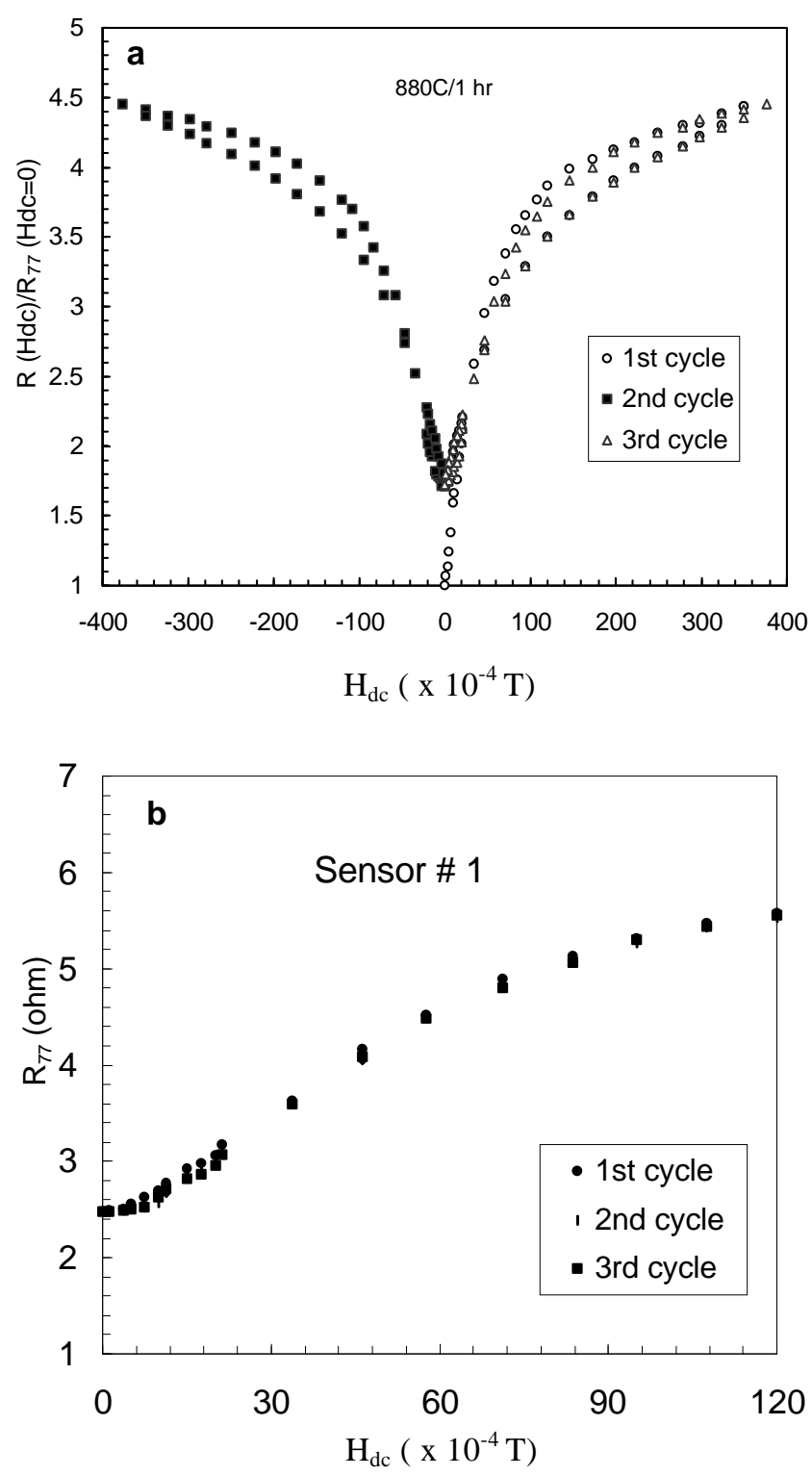

Figure 4. a. Hysteretic characteristics of the sensor \#1 at liquid nitrogen temperature for magnetic field between -40 and $+40 \mathrm{mT}$ and b. hysteretic characteristics of the sensor \#1 at liquid nitrogen temperature for magnetic field between -12 and $+12 \mathrm{mT}$.

fact that the ceramic superconductors undergo degradation in presence of moisture, water vapour etc, suitable protection of the sensors would be necessary for their reliable long term use.

\section{Conclusions}

The performance of a simple, inexpensive magnetic sensor fabricated using BPSCCO screen-printed thick film on $\mathrm{MgO}$ (100) substrate has been presented. Our results indicate that BPSCCO thick film heat treated at $880 \mathrm{C}$ for $1 \mathrm{~h}$ gives the best response to the external d.c. field. The sensor resistance at $77.4 \mathrm{~K}$ for $H_{\text {d.c. }}$ of $\pm 10 \mathrm{mT}$ (100 gauss) displays an increase of $360 \%$ over its resistance in zero field. Such a high response, along with its reproducibility against thermal cycling and zero hysteresis for field below $12 \mathrm{mT}$ provide the basis for its use as an inexpensive and sensitive magnetic field sensor $\left(<12 \times 10^{-3} \mathrm{~T}\right)$.

\section{Acknowledgement}

One of the authors (TKD) thanks the Department of Science and Technology, New Delhi, for a research grant.

\section{References}

Buckley J R, Khare N, Donaldson G B, Cochran A and Hui Z 1991 Trans. Magn. 27309

Dey T K, Chattopadhyay M K and Ghatak S K 1999 Sensors and Actuators $\mathbf{B 5 5} 222$

Dibbern U and Petersen A 1983 Electron. Comp. Appl. 5148

Gallop J C, Lilleyaman S, Langham C D, Redcliffe W J and Stewart M 1989 IEEE Trans. Magn. 25896

Gielisse P J, Niculescu H, Roy B, Jones K W, Larkin G and Hu Z 1991 Supercond. Sci. Technol. 4416

Heremans J 1993 J. Phys. D. Appl. Phys. 261149

Itoh M, Ishigaki $\mathrm{H}$ and Hida A 1989 IEEE Trans. Magn. 25 2518

Itoh M, Pavese F, Mori K, Vanolo M and Minemoto T 1997 J. Mag. Soc. Jap. 20289

Khare N, Gupta A K, Shrivastava S K, Khare S and Padmanaban V P N 1997 Meas. Sci. Technol. 829

Kirschner I, Leppavuori S, Porjesz T, Uusimaki A and Kokkamaki T 1994 Sensors and Actuators A41 58

Lenz J E 1990 Proc. IEEE 78973

Ripka P 1992 Sensors and Actuators A33 129

Rostami K R, Mantorov V V and Sukhanov A A 1998 Sensors and Actuators $\mathbf{A 6 5} 10$

Villegier J C et al 1991 IEEE Trans. Magn. 271552

Wise S A, Buckley J D and Niholt J 1993 Appl. Supercond. 1 1363

Zheng J P, Ying Q Y and Kwok H S 1990 Physica C 168322 